PION PRODUCTION MODELS AND NEUTRINO FACTORIES

\author{
Johann Collot \\ ISN de Grenoble-CNRS/IN2P3-Univ. Joseph Fourier \\ Harold G. Kirk \\ Brookhaven National Laboratory \\ Nikolai V. Mokhov \\ Fermilab
}

This research has been supported in part by the U.S. Department of Energy under contract nos. DE-ACO2-98CH10886 and DE-ACO2-76CHO30OO 


\title{
Pion Production Models and Neutrino Factories
}

\author{
Johann Collot ${ }^{\mathrm{a}}$, Harold G. Kirk ${ }^{\mathrm{b}}$, Nikolai V. Mokhov ${ }^{\mathrm{c}}$ \\ ${ }^{\mathrm{a}}$ ISN de Grenoble - CNRS/IN2P3 - Université Joseph Fourier \\ ${ }^{\mathrm{b}}$ Brookhaven National Laboratory, Upton, NY, 11973-5000 USA \\ ${ }^{\mathrm{c}}$ Fermi National Accelerator Laboratory, Batavia, IL, 60510, USA
}

\begin{abstract}
Scenarios for the building of muon colliders or storage rings suitable for the generation of robust neutrino beams call for the generation of a prodigious quantity of pions. These pions are then conducted into a decay channel where the resulting muon decay products can be collected for cooling and subsequent acceleration. Central to this concept is the design and construction of a target which will be highly efficient in producing pions of both signs while mitigating the absorption of these pions before they decay. This design effort is being facilitated by using two computer codes FLUKA and MARS. We present comparisons of the two computer codes and also present a comparison of these codes with available data.
\end{abstract}

\section{Introduction}

A key issue toward the development of a muon collider or neutrino beam based on a muon storage ring is the design of a target/capture system capable of capturing a large number of pions. These pions then proceed into a decay channel where the resultant muon decay products are harvested before being conducted into a cooling channel and then subsequently accelerated to the final energy of the facility. Central to the design of an optimum system to accomplish this task is the development of a set of tools which can be relied upon to calculate the correct pion production characteristics for various target materials and geometries under consideration. We report in this paper on the results of an investigation of two important tools available to the high-energy physics community, the current versions of the MARS(1) and FLUKA(2) Monte Carlo codes. 


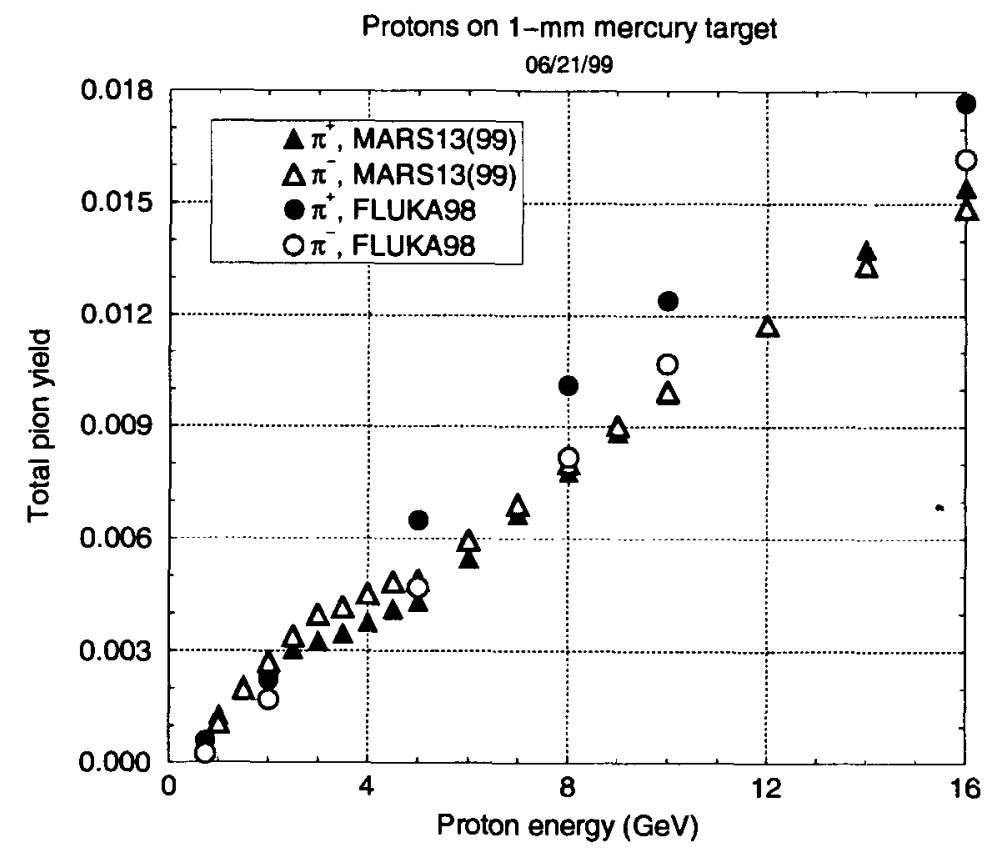

Fig. 1. Total pion yield from a thin mercury target

\section{Comparison of pion yields}

Current scenarios for muon colliders/storage rings call for copious pion production. We explore the total $\pi^{+}$and $\pi^{-}$yields resulting from proton-nucleus interactions over the range of incoming proton energies considered for candidate proton machines which could serve as drivers for a muon collider or muon storage ring facility.

We compare directly the particle production aspects of the current versions of two codes FLUKA and MARS by examining the total pion production from a thin target so that tracking and secondary production issues will not unnecessarily complicate the comparison. The $1 \mathrm{~mm}$ thick mercury target corresponds to approximately $0.7 \%$ of the nuclear inelastic interaction length. Figure 1 shows the direct comparison of the two codes. The vertical axis has been normalized to the total incident protons. We conclude that the two codes give good agreement for total pion production in the incident proton kinetic energy range of 1 to $16 \mathrm{GeV}$. The most significant discrepancy can be seen for the positive pion production in which FLUKA gives approximately $25 \%$ higher yields for incident energies greater than $4 \mathrm{GeV}$. 

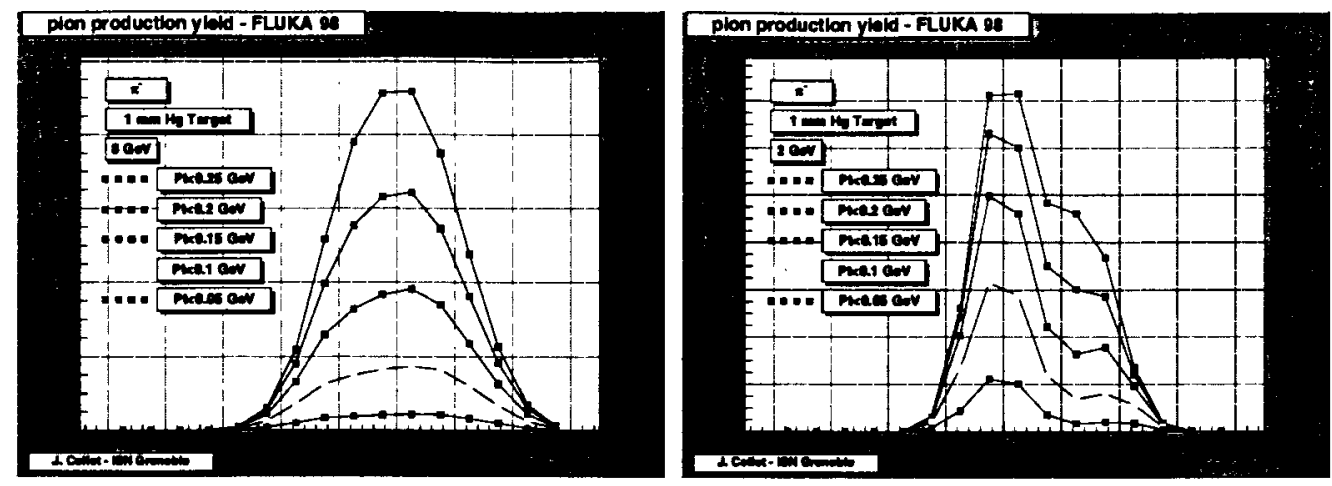

Fig. 2. FLUKA-Negative pion spectra from a thin heavy target

\section{Comparison of pion spectra}

Two methods of pion capture are currently favored, both based on the capture of forward produced low-energy pions. One scenario(3) is based on immersing the target in a high-field $(20 \mathrm{~T})$ solenoid magnet so that forward produced pions with $\mathrm{p}_{t}<220 \mathrm{MeV} / \mathrm{c}$ are captured. A second method being explored makes use of a horn system which also focuses and captures these same lowenergy pions. Consequently, it is important from the modeling point-of-view that the pion production spectra be accurately rendered.

We consider the negative pion production spectrum at two incident energies of 2 and $8 \mathrm{GeV}$. In Figure 2, we examine the results of FLUKA calculations. It is noticed that for the $2 \mathrm{GeV}$ incident proton case, a shoulder appears in the rapidity region of 1 to 2 . We show in Figure 3 the results for MARS. The MARS results are shown normalized for $10^{6}$ incoming protons and give a similar yield and spectrum for produced pions at $8 \mathrm{GeV}$ but do exhibit a different spectrum for the $2 \mathrm{GeV}$ incident proton case. The MARS result is characterized by the absence of a shoulder in the rapidity region of 1 to 2 . It should be pointed out that for low-energy pion capture, the rapidity region 0.5 to 2 is most important and hence FLUKA and MARS discrepancies in this region of phase space are of concern.

\section{Comparison with data}

In this section we compare MARS and FLUKA predictions with some data. We show in Figure 4 a comparison of total pion yield to incident proton energy over the interesting regime of 1 to $20 \mathrm{GeV}$. Here the results are depicted for proton beams incident on light, medium, and heavy nuclei. The agreement of total pion yield to the model is quite good. A comparison of the pion spectrum to model predictions is a more delicate issue because the region of the pion spectrum that most interest us, namely the low-energy pions, is least 


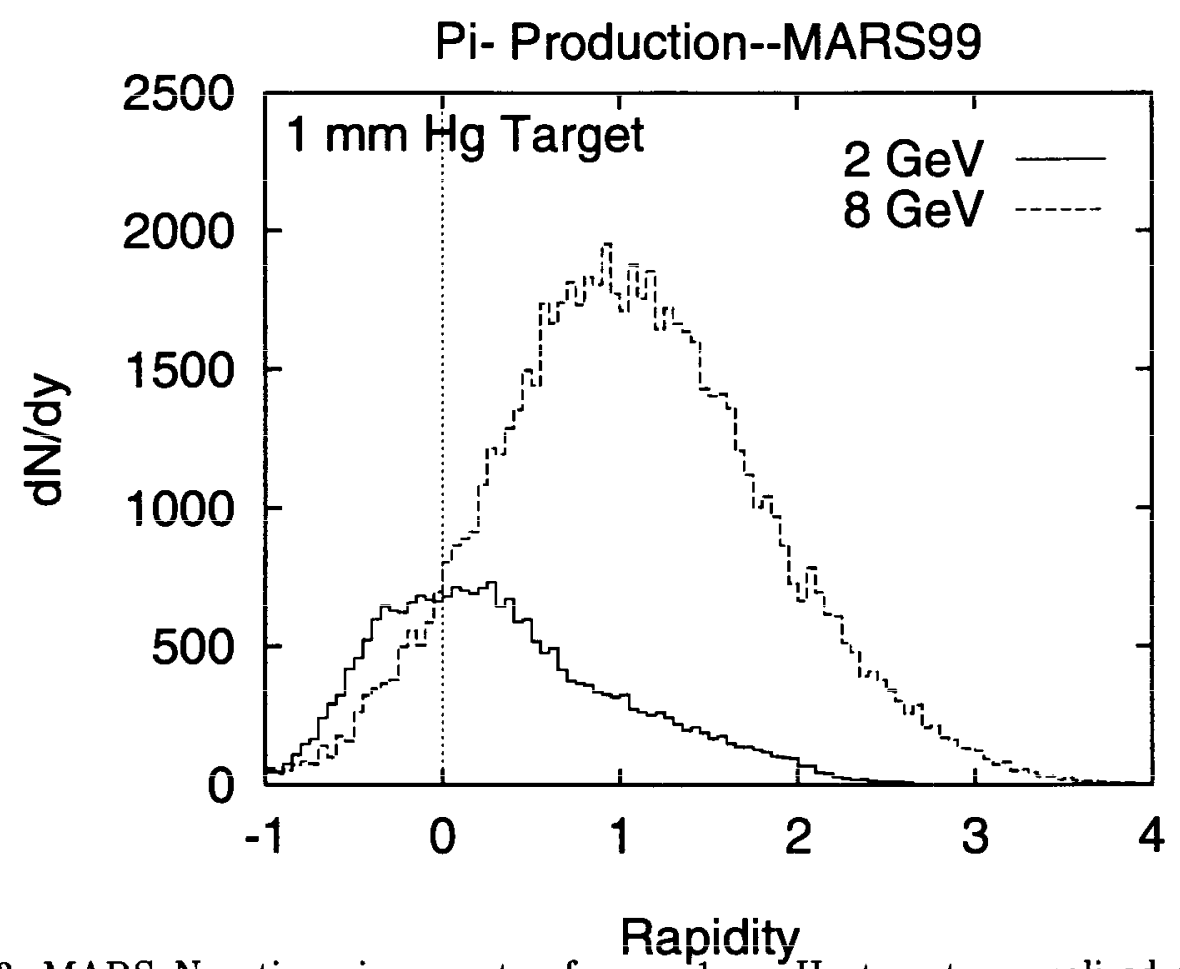

Fig. 3. MARS-Negative pion spectra from a $1 \mathrm{~mm} \mathrm{Hg}$ target normalized to $10^{6}$ incoming protons. No transverse momentum selection is imposed on the spectra.

well known. For experiments with high-energy protons incident on nuclei, the fast, forward part of the production spectra tends to be well measured but the slower high-angle produced particles tend to have flight paths outside the acceptance of the experimental apparatus. Two experiments promise to help resolve this situation. E910(4) at the Brookhaven AGS has an acceptance for forward produced particle up to $90^{\circ}$ while a recently proposed experiment at the CERN PS, P315(5), is designed to have even larger acceptances. E910 will soon report data for incident proton beams of 6,12 , and $18 \mathrm{GeV}$ on targets of $\mathrm{Be}, \mathrm{Cu}$, and $\mathrm{Au}$. P315 proposes incident proton beams from 2 to $16 \mathrm{GeV}$.

We show in Figure 5 a comparison of data with calculations with the CEM97 code(6) used in MARS as an event generator at $E<4 \mathrm{GeV}(1)$ and with the FLUKA code(2) for the cases of 3 and $4 \mathrm{GeV} / \mathrm{c}$ protons incident on aluminum nucleus. Although the agreement is quite encouraging, more data is certainly needed to understand existing differences especially at $\mathrm{E}<100 \mathrm{MeV}$.

\section{Summary}

Pion production modeling via the two codes FLUKA and MARS have been compared with the result that broad agreement between the two codes is found. Total pion yields are comparable with the two codes. An examination of pion spectra shows general agreement but some differences in detail. In 

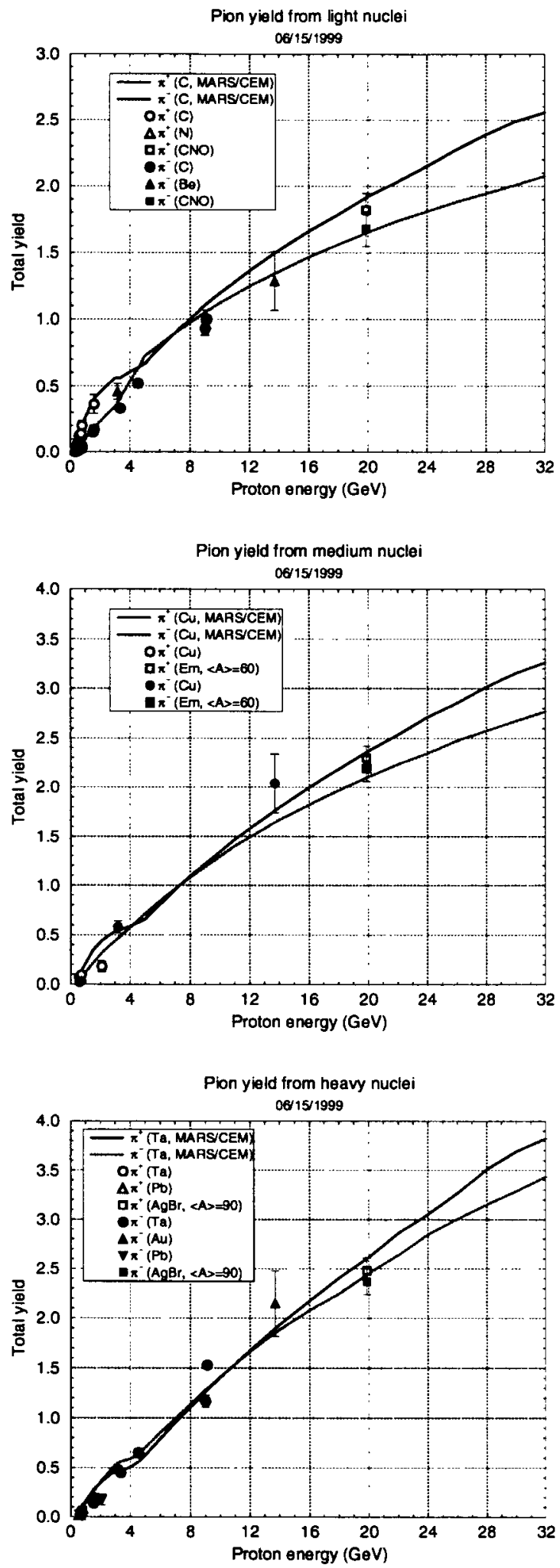

Fig. 4. MARS-Total pion yields from various targets 

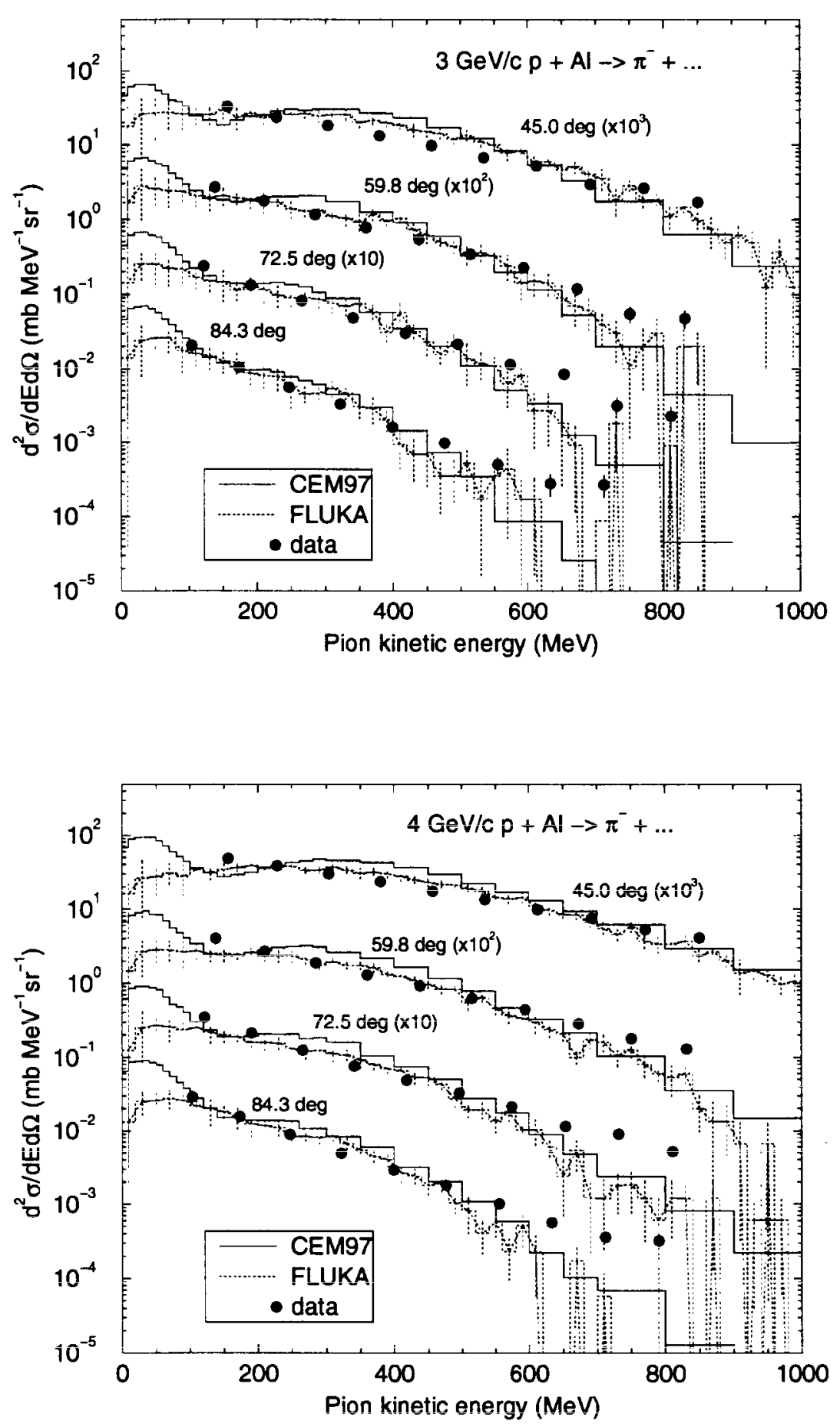

Fig. 5. Comparison of MARS(CEM) and FLUKA calculated pion spectra to data(7; 8 ) at incident proton momenta of 3 and $4 \mathrm{GeV} / \mathrm{c}$ on aluminum nucleus. (Thanks to S. Chiba, A. Ferrari and S. Mashnik for providing us with the numerical data.) 
particular the portion of phase space represented by the rapidity region 1 to 2 needs attention. Of particular concern is the sparseness of data for lowenergy pion production over a large number of nuclear species $(Z=3$ to 92$)$ and a broad range of interesting energies $(1-30 \mathrm{GeV})$. This lack of data is being addressed by two experiments, E910 at the Brookhaven AGS (6 to 18 $\mathrm{GeV}$ ) and a hadron production experiment, P315, currently being prepared for running at the CERN PS (2 to $16 \mathrm{GeV}$ ).

\section{References}

[1] N. V. Mokhov, "The mars Code System User Guide, Version 13(95)", Fermilab-FN-628 (1995); N. V. Mokhov et al., Fermilab-Conf-98/379 (1998); LANL Report LA-UR-98-5716 (1998); nucl-th/9812038 v2 16 Dec 1998; http://www-ap.fnal.gov/MARS/.

[2] A. Fassø, A. Ferrari, J. Ranft, P.R. Sala, New developments in FLUKA modeling hadronic and EM interactions, Proc. 3rd Workshop on Simulating Accelerator Radiation Environments, KEK, Tsukuba, Japan 7-9 May 1997 Ed. H. Hirayama, KEK Proceedings 97-5, p. 32-43. A. Ferrari, and P.R. Sala, The Physics of High Energy Reactions, in Proceedings of the Workshop on Nuclear Reaction Data and Nuclear Reactors Physics, Design and Safety, International Centre for Theoretical Physics, Miramare-Trieste, Italy, 15 April-17 May 1996, edited by A. Gandini and G. Reffo, World Scientific, p. 424 (1998).

[3] H.G. Kirk, Proceedings of the 1999 Particle Accelerator Conference, New York (1999) 3029.

[4] Experiment E910 at the BNL AGS http://www.nevis.columbia.edu/heavyion/e910/

[5] Proposal P315 at the CERN PS http://choruswww.cern.ch/ panman/hpeproposal.ps

[6] S. G. Mashnik and A. J. Sierk, Proc. of the Fourth Workshop on Simulating Accelerator Radiation Environments (SARE4), Knoxville, TN, September 14-16, 1998, p. 29.

[7] H. En'yo et al., Phys. Lett., B159 (1985) 1.

[8] K. Niita et al., Phys. Rev., C52 (1995) 2620. 